\title{
A Hopf Bifurcation in a Free Boundary Problem with an Inhomogeneity
}

\author{
YoonMee Ham \\ Communicated by Charles Li, received October 21, 2004
}

\begin{abstract}
We shall consider a two-component reaction-diffusion system with spatial inhomogeneous media. In this paper, we shall explore the dynamics of interfaces in the problem with spatial inhomogeneous media in order to investigate the existence of time periodic solutions as a parameter vary and to examine the effect of a spatial inhomogeneity.
\end{abstract}

\section{Contents}

1. Introduction 59

2. Regularized equation $\quad 60$

3. Existence of steady-states and Linearized equation 61

4. Effects of an inhomogeneity for a Hopf bifurcation 62

References $\quad 68$

\section{Introduction}

Nonlinear reaction-diffusion equation models have been used to study impulses propagating in nerve axon [5], to describe pattern formation [17] and to model spiral waves in the Belousov-Zhabotinsky reaction [23]. In most models for such waves, the medium is assumed to be homogeneous. The effect of an inhomogeneous diffusion process was studied for one component system in $[\mathbf{1 8}, \mathbf{2 4}]$ and a two component system close to a singular limit in $[\mathbf{6}, \mathbf{7}]$. The most simple but substantial model is given by the following reaction-diffusion equations with a spatial inhomogeneity $[\mathbf{1}, \mathbf{1 0}, \mathbf{1 9}, \mathbf{2 1}]$ :

$$
\left\{\begin{array}{l}
\varepsilon \sigma u_{t}-\varepsilon^{2} u_{x x}=H\left(u-a_{0}\right)-u-v+\kappa \equiv f(u, v)+\kappa \\
v_{t}-v_{x x}=u-\mu v \equiv g(u, v), \quad x \in(-\infty, \infty), t>0
\end{array}\right.
$$

1991 Mathematics Subject Classification. 35R35, 35B32, 35B25, 35K22, 35K57.

Key words and phrases. free boundary problem, inhomogeneity, Hopf bifurcation.

The present work was supported by the Research Foundation at Kyonggi University in 2004. 
where $\varepsilon, \sigma, \mu$ are all positive constants, $0<a_{0}<1$ and $H(z)$ is a Heaviside step function $([\mathbf{1 1}])$. The equation (1) represents a basic model of bistable medium which is relevant for fertilization calcium waves [10] and current density dynamics in large area bistable semiconductor systems $[\mathbf{3}, \mathbf{2 2}]$.

When $\varepsilon$ is sufficiently small and $\kappa=0$ in the equation (1), a motionless localized solution $[\mathbf{9}]$ and a spatially periodic solution $[\mathbf{1 2}, \mathbf{1 6}]$ can be formed. In this cases, the authors in $[\mathbf{2}, \mathbf{1 5}, \mathbf{2 0}]$ showed that in the limit the stationary solutions of this system, being smooth, exhibits an abrupt change but continuously differentiable transition at the location of the internal layers. This transition takes place with in an $x$-interval of length $O(\varepsilon)$ and converges to interfacial curves in $x, t$-space as $\varepsilon \downarrow 0$. When $\varepsilon=0$ in the first equation (1), an analysis of the dynamics of this process has been shown (see for example $[\mathbf{2}, \mathbf{1 3}, \mathbf{1 4}, \mathbf{1 5}]$ ) to lead a free boundary problem consisting of the initial-boundary value problem

(2)

$$
\left\{\begin{array}{l}
v_{t}=v_{x x}-(\mu+1) v+H\left(x-\eta_{+}\right)-H\left(x-\eta_{-}\right)+\kappa,(x, t) \in \Omega^{+}(t) \cup \Omega^{-}(t) \\
v(x, 0)=v_{0}(x) \\
v(-\infty, t)=0=v(\infty, t), t>0 \\
\eta_{ \pm}^{\prime}(t)= \pm C\left(v\left(\eta_{ \pm}(t)\right)\right), t>0 \\
\eta_{ \pm}(0)= \pm \eta_{0}, \eta_{0} \in(0, \infty)
\end{array}\right.
$$

where $\eta \pm(t)$ are interfaces and the domains are $\Omega^{+}(t)=\left\{(x, t): \eta_{-}(t)<x<\right.$ $\left.\eta_{+}(t), t>0\right\}$ and $\Omega^{-}(t)=\left\{(x, t): x<\eta_{-}(t), x>\eta_{+}(t), t>0\right\}$. From $[\mathbf{2}, \mathbf{8}, \mathbf{1 5}$, 22], the trajectory with a unique value of $C=C\left(v_{0}\right)$ exists and the velocity of the interface is a continuously differentiable function defined on an interval $I:=$ $\left(-a_{0}, 1-a_{0}\right)$ and thus the velocity of the interface can be normalized by

$$
C(z)=\frac{2 z-1+2 a_{0}-2 \kappa}{\sigma \sqrt{\left(z+a_{0}-\kappa\right)\left(1-a_{0}+\kappa-z\right)}} .
$$

The purpose of this paper is to explore the dynamics of interfaces in the problem (2) in order to examine the effects of the a spatial inhomogeneity $\kappa$.

The organization of the paper is as follows. In section 2 we show the existence of the periodic solutions and the bifurcation of the interface problem as the parameter $\sigma$ and $\kappa$ vary by invoking the regular setting of the problem (2). In section 3 , we examine the occurences of Hopf bifurcation as the parameter $\sigma$ varies and investigate the effects of the spatial inhomogeneity.

\section{Regularized equation}

For the application of semigroup theory to (2), we choose the space $X:=L_{2}(\mathbb{R})$ with norm $\|\cdot\|_{2}$ and let $A$ be a differential operator $-\frac{d^{2}}{d x^{2}}+\mu+1$ with the domain $D(A)=\left\{v \in H^{2,2}((-\infty, \infty)): v(-\infty)=v(\infty)=0\right\}$.

DEFinition 2.1. We call $\left(v, \eta_{-}, \eta_{+}\right)$a solution of (2), if it satisfies the following natural properties: There exists $T>0$ such that $v(x, t)$ is defined for $(x, t) \in$ $\mathbb{R} \times[0, T), \eta_{ \pm} \in \mathbb{R}$ and $v\left(\eta_{ \pm}(t), t\right) \in I$ for $t \in[0, T)$,

a) $v(\cdot, t) \in C^{1}(\mathbb{R})$ for $t>0$ with $v( \pm \infty, t)=0$,

b) $\eta_{-}, \eta_{+} \in C^{0}([0, T)) \cap C^{1}((0, T))$ with $\eta_{ \pm}(0)= \pm \eta_{0}, \eta_{0} \in(0, \infty)$,

c) $(A v)(x, t)$ and $v_{t}(x, t)$ exist for $x \in \mathbb{R} \backslash\left\{\left\{\eta_{-}(t)\right\} \cup\left\{\eta_{+}(t)\right\}\right\}$ and $t \in(0, T)$, 
d) $t \mapsto v(\cdot, t) \in C^{0}([0, T), X)$ with $v(\cdot, 0)=v_{0} \in X$ and

e) $v, \eta_{-}$and $\eta_{+}$solve the differential equation for $t \in(0, T)$ and $x \in \mathbb{R} \backslash$ $\left\{\left\{\eta_{-}(t)\right\} \cup\left\{\eta_{+}(t)\right\}\right\}$.

We define $g: \mathbb{R}^{3} \longrightarrow \mathbb{R}$,

$g\left(x, \eta_{-}, \eta_{+}\right):=A^{-1}\left(H\left(\cdot-\eta_{-}\right)-H\left(\cdot-\eta_{+}\right)+\kappa\right)(x)=\int_{\eta_{-}}^{\eta_{+}} G(x, y) d y+\kappa \int_{\mathbb{R}} G(x, y) d y$,

where $G: \mathbb{R}^{2} \rightarrow \mathbb{R}$ is a Green's function of $A$ satisfying the Dirichlet boundary conditions. Applying the transformation $u(t)(x)=v(x, t)-g\left(x, \eta_{-}(t), \eta_{+}(t)\right)$ then we obtain an equivalent abstract evolution equation of (2) :

$$
\left\{\begin{array}{l}
\frac{d}{d t}\left(u, \eta_{-}, \eta_{+}\right)+\widetilde{A}\left(u, \eta_{-}, \eta_{+}\right)=f\left(u, \eta_{-}, \eta_{+}\right) \\
\left(u, \eta_{-}, \eta_{+}\right)(0)=\left(u_{0}(x),-\eta_{0}, \eta_{0}\right)
\end{array}\right.
$$

where $\widetilde{A}$ is a $3 \times 3$ matrix whose $(1,1)$-entry is an operator $A$ and all the others are zero. The nonlinear forcing term $f$ is

$f\left(u, \eta_{-}, \eta_{+}\right)=\left(\begin{array}{c}-C\left(u\left(\eta_{-}\right)+\gamma\left(\eta_{-}, \eta_{+}\right)\right) G\left(x, \eta_{-}\right)-C\left(u\left(\eta_{+}\right)+\zeta\left(\eta_{-}, \eta_{+}\right)\right) G\left(x, \eta_{+}\right) \\ -C\left(u\left(\eta_{-}\right)+\gamma\left(\eta_{-}, \eta_{+}\right)\right) \\ C\left(u\left(\eta_{+}\right)+\zeta\left(\eta_{-}, \eta_{+}\right)\right)\end{array}\right)$

where the functions $\gamma: \mathbb{R} \times \mathbb{R} \longrightarrow \mathbb{R}$ and $\zeta: \mathbb{R} \times \mathbb{R} \longrightarrow \mathbb{R}$ are defined by $\gamma\left(\eta_{-}, \eta_{+}\right):=$ $g\left(\eta_{-}, \eta_{-}, \eta_{+}\right)$and $\zeta\left(\eta_{-}, \eta_{+}\right):=g\left(\eta_{+}, \eta_{-}, \eta_{+}\right)$.

The well posedness of solutions are shown in [4] applying the semigroup theory using domains of fractional powers $\alpha \in(3 / 4,1]$ of $A$ and $\widetilde{A}$. Moreover, they obtained that the nonlinear term $f$ is a continuously differentiable function from $W \cap D\left(\widetilde{A}^{\alpha}\right)$ to $D(\widetilde{A})$ where $D(\widetilde{A})=D(A) \times \mathbb{R} \times \mathbb{R}$ and

$W:=\left\{\left(u, \eta_{-}, \eta_{+}\right) \in C^{1}(\mathbb{R}) \times \mathbb{R} \times \mathbb{R}: u\left(\eta_{-}\right)+\gamma\left(\eta_{-}, \eta_{+}\right) \in I, u\left(\eta_{+}\right)+\zeta\left(\eta_{-}, \eta_{+}\right) \in I\right\}$

$$
\subset_{\text {open }} C^{1}(\mathbb{R}) \times \mathbb{R} \times \mathbb{R} \text {. }
$$

\section{Existence of steady-states and Linearized equation}

In this section, we shall examine the stationary solutions of (3) and shall study the dependency of $\kappa$ on the existence of solutions. We now consider the stationary problem of $(3)$ for $\left(u^{*}, \eta_{-}^{*}, \eta_{+}^{*}\right) \in D(\widetilde{A}) \cap W$. This system is equivalent to the pair of equations

$$
u^{*}=0, C\left(\gamma\left(\eta_{-}^{*}, \eta_{+}^{*}\right)\right)=0 \text { and } C\left(\zeta\left(\eta_{-}^{*}, \eta_{+}^{*}\right)\right)=0
$$

which implies that

$$
\frac{1}{2}-a_{0}-\gamma\left(\eta_{-}^{*}, \eta_{+}^{*}\right)+\kappa=0 \text { and } \frac{1}{2}-a_{0}-\zeta\left(\eta_{-}^{*}, \eta_{+}^{*}\right)+\kappa=0 .
$$

Proposition 3.1. Suppose that $0<\frac{1}{2}-a_{0}+\frac{\mu \kappa}{\mu+1}<\frac{1}{2(\mu+1)}$, then the equation (3) has at least one stationary solutions $\left(0, \eta_{-}^{*}, \eta_{+}^{*}\right)$ for all $\sigma \neq 0$ satisfying that $\eta_{+}^{*}-\eta_{-}^{*}=\frac{1}{\sqrt{\mu+1}} \ln \left(\frac{1}{2(\mu+1) a_{0}-\mu-2 \mu \kappa}\right)$. 
The linearization of $f$ at the stationary solution $\left(0, \eta_{-}^{*}, \eta_{+}^{*}\right)$ is

$$
\begin{aligned}
& \operatorname{Df}\left(0, \eta_{-}^{*}, \eta_{+}^{*}\right)\left(\hat{u}, \hat{\eta}_{-}, \hat{\eta}_{+}\right) \\
& =\left(\begin{array}{c}
\frac{4}{\sigma}\left(\hat{u}\left(\eta_{-}^{*}\right)+\gamma_{\eta_{-}}\left(\eta_{-}^{*}, \eta_{+}^{*}\right) \hat{\eta}_{-}+\gamma_{\eta_{+}}\left(\eta_{-}^{*}, \eta_{+}^{*}\right) \hat{\eta}_{+}\right) G\left(\cdot, \eta_{-}^{*}\right) \\
+\frac{4}{\sigma}\left(\hat{u}\left(\eta_{+}^{*}\right)+\zeta_{\eta_{-}}\left(\eta_{-}^{*}, \eta_{+}^{*}\right) \hat{\eta}_{-}+\zeta_{\eta_{+}}\left(\eta_{-}^{*}, \eta_{+}^{*}\right) \hat{\eta}_{+}\right) G\left(\cdot, \eta_{+}^{*}\right) \\
\frac{4}{\sigma}\left(\hat{u}\left(\eta_{-}^{*}\right)+\gamma_{\eta_{-}}\left(\eta_{-}^{*}, \eta_{+}^{*}\right) \hat{\eta}_{-}+\gamma_{\eta_{+}}\left(\eta_{-}^{*}, \eta_{+}^{*}\right) \hat{\eta}_{+}\right) \\
-\frac{4}{\sigma}\left(\hat{u}\left(\eta_{+}^{*}\right)+\zeta_{\eta_{-}}\left(\eta_{-}^{*}, \eta_{+}^{*}\right) \hat{\eta}_{-}+\zeta_{\eta_{+}}\left(\eta_{-}^{*}, \eta_{+}^{*}\right) \hat{\eta}_{+}\right)
\end{array}\right) .
\end{aligned}
$$

The pair $\left(0, \eta_{-}^{*}, \eta_{+}^{*}\right)$ corresponds to a unique steady state $\left(v^{*}, \eta_{-}^{*}, \eta_{+}^{*}\right)$ of (2) for $\sigma \neq 0$ with $v^{*}(x)=g\left(x, \eta_{-}^{*}, \eta_{+}^{*}\right)$.

Proof. From (4) and (5), the steady states $\left(0, \eta_{-}^{*}, \eta_{+}^{*}\right)$ are solutions of the following equation :

$$
\frac{1}{2}-a_{0}+\kappa-\gamma\left(\eta_{-}, \eta_{+}\right)=0 .
$$

The equation (6) implies that $\frac{1}{2(\mu+1)}\left(1-e^{-\sqrt{\mu+1}\left(\eta_{+}-\eta_{-}\right)}\right)+\frac{\kappa}{\mu+1}=\frac{1}{2}-a_{0}+\kappa$. Thus $\left(\eta_{-}^{*}, \eta_{+}^{*}\right)$ satisfies that $\eta_{+}^{*}-\eta_{-}^{*}=\frac{1}{\sqrt{\mu+1}} \ln \left(\frac{1}{2(\mu+1) a_{0}-\mu-2 \mu \kappa}\right)$ if $0<\frac{1}{2}-a_{0}+\frac{\mu \kappa}{\mu+1}<$ $\frac{1}{2(\mu+1)}$.

The formula for $\operatorname{Df}\left(0, \eta_{-}^{*}, \eta_{+}^{*}\right)$ follows from the relation $C^{\prime}\left(0, \eta_{-}^{*}, \eta_{+}^{*}\right)=4 / \sigma$ and Lemma 4 in [4]. The corresponding steady state $\left(v^{*}, \eta_{-}^{*}, \eta_{+}^{*}\right)$ for $(2)$ is obtained using the transformation and Proposition 7 in $[4]$.

We state the linearized eigenvalue problem of $(3)$ :

$$
-\widetilde{A}\left(u, \eta_{-}, \eta_{+}\right)+\tau B\left(u, \eta_{-}, \eta_{+}\right)=\lambda\left(u, \eta_{-}, \eta_{+}\right)
$$

which is equivalent to

$$
\left\{\begin{aligned}
(A+\lambda) u & =\tau\left(u\left(\eta_{-}^{*}\right)+\gamma_{\eta_{-}} \eta_{-}+\gamma_{\eta_{+}} \eta_{+}\right) G\left(\cdot, \eta_{-}^{*}\right) \\
& +\tau\left(u\left(\eta_{+}^{*}\right)+\gamma_{\eta_{-}} \eta_{-}+\gamma_{\eta_{+}} \eta_{+}\right) G\left(\cdot, \eta_{+}\right) \\
\lambda \eta_{-} & =\tau\left(u\left(\eta_{-}^{*}\right)+\gamma_{\eta_{-}} \eta_{-}+\gamma_{\eta_{+}} \eta_{+}\right) \\
-\lambda \eta_{+} & =\tau\left(u\left(\eta_{+}^{*}\right)+\gamma_{\eta_{-}} \eta_{-}+\gamma_{\eta_{+}} \eta_{+}\right)
\end{aligned}\right.
$$

where $\gamma_{\eta_{-}}:=\gamma_{\eta_{-}}\left(\eta_{-}^{*}, \eta_{+}^{*}\right), \gamma_{\eta_{+}}:=\gamma_{\eta_{+}}\left(\eta_{-}^{*}, \eta_{+}^{*}\right)$ and $\tau=4 / \sigma$.

\section{Effects of an inhomogeneity for a Hopf bifurcation}

DEFINITION 4.1. Under the assumptions of Proposition 3.1, define (for $1 \geq$ $\alpha>3 / 4)$ the linear operator $B$ from $\tilde{X}^{\alpha}$ to $\tilde{X}$

$$
B:=\frac{\sigma}{4} \operatorname{Df}\left(0, \eta_{-}^{*}, \eta_{+}^{*}\right) .
$$

We then define $\left(0, \eta_{-}^{*}, \eta_{+}^{*}\right)$ to be a Hopf point for (3) if and only if there exists an $\epsilon_{0}>0$ and $a C^{1}$-curve

$$
\left(-\epsilon_{0}+\tau^{*}, \tau^{*}+\epsilon_{0}\right) \mapsto(\lambda(\tau), \phi(\tau)) \in \mathbb{C} \times \tilde{X}_{\mathbf{C}}
$$

$\left(Y_{\mathbf{C}}\right.$ denotes the complexification of the real space $\left.Y\right)$ of eigendata for $-\widetilde{A}+\tau B$ with

(i) $(-\widetilde{A}+\tau B)(\phi(\tau))=\lambda(\tau) \phi(\tau), \quad(-\widetilde{A}+\tau B)(\overline{\phi(\tau))}=\overline{\lambda(\tau)} \overline{\phi(\tau)}$; 
(ii) $\lambda\left(\tau^{*}\right)=i \beta$ with $\beta>0$;

(iii) $\operatorname{Re}(\lambda) \neq 0$ for all $\lambda \in \sigma\left(-\widetilde{A}+\tau^{*} B\right) \backslash\{ \pm i \beta\}$;

(iv) $\operatorname{Re} \lambda^{\prime}\left(\tau^{*}\right) \neq 0$ (transversality).

We shall show that the steady states become a Hopf point under the same condition in Proposition 3.1.

TheOREM 4.2. Assume $0<\frac{1}{2}-a_{0}+\frac{\mu \kappa}{\mu+1}<\frac{1}{2(\mu+1)}$, the operator $-\widetilde{A}+\tau^{*} B$ as a unique pair $\{ \pm i \beta\}$ of purely imaginary eigenvalues for some $\tau^{*}>0$. Then $\left(0, \eta_{-}^{*}, \eta_{+}^{*}, \tau^{*}\right)$ is a Hopf point for (3).

Proof. We assume without loss of generality that $\beta>0$, and $\phi^{*}$ is the (normalized) eigenfunction of $-\widetilde{A}+\tau^{*} B$ with eigenvalue $i \beta$. We have to show that $\left(\phi^{*}, i \beta\right)$ can be extended to a $C^{1}$-curve $\tau \mapsto(\phi(\tau), \lambda(\tau))$ of eigendata for $-\widetilde{A}+\tau B$ with $\operatorname{Re}\left(\lambda^{\prime}\left(\tau^{*}\right)\right) \neq 0$.

For this let $\phi^{*}=\left(\psi_{0}, \varrho_{0}, \rho_{0}\right) \in D(A) \times \mathbb{R} \times \mathbb{R}$. First, we see that $\varrho_{0} \neq 0$ and $\rho_{0} \neq 0$, for otherwise, if $\varrho_{0}=0=\rho_{0}$ then, by $(7),(A+i \beta) \psi_{0}=i \beta\left(\varrho_{0} G\left(\cdot, \eta_{-}^{*}\right)-\right.$ $\left.\rho_{0} G\left(\cdot, \eta_{+}^{*}\right)\right)=0$, which is not possible because $A$ is symmetric. So without loss of generality, let $\varrho_{0}=1$. Then $E\left(\psi_{0}, \rho_{0}, i \beta, \tau^{*}\right)=0$ by $(7)$, where

$E: D(A)_{\mathbf{C}} \times \mathbb{R} \times \mathbb{C} \times \mathbb{R} \longrightarrow X_{\mathbf{C}} \times \mathbb{C} \times \mathbb{C}$,

$E\left(u, \eta_{+}, \lambda, \tau\right)=$

$$
\left(\begin{array}{c}
(A+\lambda) u-\tau\left(u\left(\eta_{-}^{*}\right)+\gamma_{\eta_{+}}\left(\eta_{+}-1\right)\right) G\left(\cdot, \eta_{-}^{*}\right)-\tau\left(u\left(\eta_{+}^{*}\right)+\gamma_{\eta_{+}}\left(\eta_{+}-1\right)\right) G\left(\cdot, \eta_{+}^{*}\right) \\
\lambda-\tau\left(u\left(\eta_{-}^{*}\right)+\gamma_{\eta_{+}}\left(\eta_{+}-1\right)\right) \\
-\lambda \eta_{+}-\tau\left(u\left(\eta_{+}^{*}\right)+\gamma_{\eta_{+}}\left(\eta_{+}-1\right)\right)
\end{array}\right)
$$

since $\gamma_{\eta_{-}}\left(\eta_{-}^{*}, \eta_{+}^{*}\right)=-\gamma_{\eta_{+}}\left(\eta_{-}^{*}, \eta_{+}^{*}\right)$. The equation $E\left(u, \eta_{+}, \lambda, \tau\right)=0$ is equivalent to $\lambda$ being an eigenvalue of $-\widetilde{A}+\tau B$ with eigenfunction $\left(u, 1, \eta_{+}\right)$. We shall here apply the implicit function theorem to $E$, and therefore have to check that $E$ is $C^{1}$ and that

$$
D_{\left(u, \eta_{+}, \lambda\right)} E\left(\psi_{0}, \rho_{0}, i \beta, \tau^{*}\right) \in L\left(D(A)_{\mathbf{C}} \times \mathbb{R} \times \mathbb{C} \times \mathbb{R}, X_{\mathbf{C}} \times \mathbb{C} \times \mathbb{C}\right)
$$

is an isomorphism. It is easy to see that $E$ is $C^{1}$. In addition, the mapping

$$
\begin{gathered}
D_{\left(u, \eta_{+}, \lambda\right)} E\left(\psi_{0}, \rho_{0}, i \beta, \tau^{*}\right)\left(\hat{u}, \hat{\eta}_{+}, \hat{\lambda}\right)= \\
\left(\begin{array}{c}
(A+i \beta) \hat{u}-\tau^{*}\left(\hat{u}\left(\eta_{-}^{*}\right)+\gamma_{\eta_{+}} \hat{\eta}_{+}\right) G\left(\cdot, \eta_{-}^{*}\right)-\tau^{*}\left(\hat{u}\left(\eta_{+}^{*}\right)+\gamma_{\eta_{+}} \hat{\eta}_{+}\right) G\left(\cdot, \eta_{+}^{*}\right)+\hat{\lambda} \psi_{0} \\
\hat{\lambda}-\tau^{*}\left(\hat{u}\left(\eta_{-}^{*}\right)+\gamma_{\eta_{+}} \hat{\eta}_{+}\right) \\
-\hat{\lambda} \rho_{0}-i \beta \hat{\eta}_{+}-\tau^{*}\left(\hat{u}\left(\eta_{+}^{*}\right)+\gamma_{\eta_{+}} \hat{\eta}_{+}\right)
\end{array}\right)
\end{gathered}
$$

is a compact perturbation of the mapping

$$
\left(\hat{u}, \hat{\eta}_{+}, \hat{\lambda}\right) \longmapsto\left((A+i \beta) \hat{u}, \hat{\eta}_{+}, \hat{\lambda}\right)
$$

which is invertible. Thus $D_{\left(u, \eta_{+}, \lambda\right)} E\left(\psi_{0}, \rho_{0}, i \beta, \tau^{*}\right)$ is a Fredholm operator of index 0 . Therefore in order to verify (8), it suffices to show that the system

$$
D_{\left(u, \eta_{+}, \lambda\right)} E\left(\psi_{0}, \rho_{0}, i \beta, \tau^{*}\right)\left(\hat{u}, \hat{\eta}_{+}, \hat{\lambda}\right)=0
$$


which is equivalent to

$$
\left\{\begin{aligned}
(A+i \beta) \hat{u}+\hat{\lambda} \psi_{0} & =\tau^{*}\left(\hat{u}\left(\eta_{-}^{*}\right)+\gamma_{\eta_{+}} \hat{\eta}_{+}\right) G\left(\cdot, \eta_{-}^{*}\right)+\tau^{*}\left(\hat{u}\left(\eta_{+}^{*}\right)\right. \\
& \left.+\gamma_{\eta_{+}} \hat{\eta}_{+}\right) G\left(\cdot, \eta_{+}^{*}\right) \\
\hat{\lambda} & =\tau^{*}\left(\hat{u}\left(\eta_{-}^{*}\right)+\gamma_{\eta_{+}} \hat{\eta}_{+}\right) \\
-\hat{\lambda} \rho_{0}-i \beta \hat{\eta}_{+} & =\tau^{*}\left(\hat{u}\left(\eta_{+}^{*}\right)+\gamma_{\eta_{+}} \hat{\eta}_{+}\right)
\end{aligned}\right.
$$

necessarily implies that $\hat{u}=0, \hat{\eta}_{+}=0$ and $\hat{\lambda}=0$. We define $\psi_{1}:=\psi_{0}-G\left(\cdot, \eta_{-}^{*}\right)+$ $G\left(\cdot, \eta_{+}^{*}\right) \rho_{0}$ then the first equation of $(9)$ is given by

$$
(A+i \beta) \hat{u}+\hat{\lambda} \psi_{1}=-i \beta G\left(\cdot, \eta_{+}^{*}\right) \hat{\eta}_{+} .
$$

On the other hand, since $E\left(\psi_{0}, \rho_{0}, i \beta, \tau^{*}\right)=0$, we have

$$
(A+i \beta) \psi_{0}=i \beta\left(G\left(\cdot, \eta_{-}^{*}\right)-G\left(\cdot, \eta_{+}^{*}\right) \rho_{0}\right)
$$

and $\psi_{1}$ is a solution to the equation

$$
(A+i \beta) \psi_{1}=-\delta_{\eta_{-}^{*}}+\delta_{\eta_{+}^{*}} \rho_{0}
$$

and

$$
\begin{aligned}
i \beta & =\tau^{*}\left(\psi_{1}\left(\eta_{-}^{*}\right)+G\left(\eta_{-}^{*}, \eta_{-}^{*}\right)-G\left(\eta_{-}^{*}, \eta_{+}^{*}\right) \rho_{0}+\gamma_{\eta_{+}}\left(\rho_{0}-1\right)\right) \\
-i \beta \rho_{0} & =\tau^{*}\left(\psi_{1}\left(\eta_{+}^{*}\right)+G\left(\eta_{+}^{*}, \eta_{-}^{*}\right)-G\left(\eta_{+}^{*}, \eta_{+}^{*}\right) \rho_{0}+\gamma_{\eta_{+}}\left(\rho_{0}-1\right)\right) .
\end{aligned}
$$

From these equations,

$$
\tau^{*} \operatorname{Im}\left(\psi_{1}\left(\eta_{-}^{*}\right)-\psi_{1}\left(\eta_{+}^{*}\right) \rho_{0}\right)=\beta\left(1+\rho_{0}^{2}\right) .
$$

Equation (11) implies that

$$
-\overline{\psi_{1}\left(\eta_{-}^{*}\right)}+\overline{\psi_{1}\left(\eta_{+}^{*}\right)} \rho_{0}=\int_{\mathbb{R}}\left|A^{1 / 2} \psi_{1}\right|^{2}+i \beta \int_{\mathbb{R}}\left|\psi_{1}\right|^{2},
$$

so that

$$
\operatorname{Im}\left(\psi_{1}\left(\eta_{-}^{*}\right)-\psi_{1}\left(\eta_{+}^{*}\right) \rho_{0}\right)=\beta \int_{\mathbb{R}}\left|\psi_{1}\right|^{2} .
$$

Hence we have

$$
\int_{\mathbb{R}}\left|\psi_{1}\right|^{2}=\frac{1}{\tau^{*}}\left(1+\rho_{0}^{2}\right) .
$$

From (11), we now can then calculate $\hat{u}\left(\eta_{ \pm}^{*}\right)$ as $\int_{\mathbb{R}}(A+i \beta) \hat{u} \psi_{1}=-\hat{u}\left(\eta_{-}^{*}\right)+\hat{u}\left(\eta_{+}^{*}\right) \rho_{0}$, which together with (9), (10) and (14) implies that

$$
\begin{aligned}
& \hat{\lambda} \int \psi_{1}^{2}+i \beta \hat{\eta}_{+} \int G\left(x, \eta_{+}^{*}\right) \psi_{1}(x) d x=\hat{u}\left(\eta_{-}^{*}\right)-\hat{u}\left(\eta_{+}^{*}\right) \rho_{0} \\
& =\hat{\lambda} \frac{1+\rho_{0}^{2}}{\tau^{*}}-\psi_{0}\left(\eta_{+}^{*}\right) \hat{\eta}_{+}=\hat{\lambda} \int_{\mathbb{R}}\left|\psi_{1}\right|^{2}-\psi_{0}\left(\eta_{+}^{*}\right) \hat{\eta}_{+} .
\end{aligned}
$$

Since from (11)

$$
\begin{aligned}
i \beta \int G\left(x, \eta_{+}^{*}\right) \psi_{1}(x) d x= & -\int A \psi_{1}(x) G\left(x, \eta_{+}^{*}\right) d x \\
& -G\left(\eta_{-}^{*}, \eta_{+}^{*}\right)+G\left(\eta_{+}^{*}, \eta_{+}^{*}\right) \rho_{0}=-\psi_{0}\left(\eta_{+}^{*}\right),
\end{aligned}
$$


and thus $i \beta \int G\left(x, \eta_{+}^{*}\right) \psi_{1}(x) d x=-\psi_{0}\left(\eta_{+}^{*}\right)$. Hence we have

$$
\hat{\lambda}\left(\int_{\mathbb{R}}\left(\psi_{1}^{2}-\left|\psi_{1}\right|^{2}\right)\right)=0
$$

which implies that $\hat{\lambda}=0$.

In order to show that $\hat{u}=0$ and $\hat{\eta}_{+}=0$ we multiply $\overline{\hat{u}}$ and $G\left(\cdot, \eta_{+}^{*}\right)$ to $(10)$, then we obtain

$$
\begin{aligned}
\int\left|A^{1 / 2} \hat{u}\right|^{2}+i \beta \int|\hat{u}|^{2} & =-i \beta \hat{\eta}_{+} \int G\left(x, \eta_{+}^{*}\right) \overline{\hat{u}(x)} d x \\
& =-\overline{\hat{u}\left(\eta_{+}^{*}\right)} \hat{\eta}_{+}+i \beta\left(\hat{\eta}_{+}\right)^{2} \int G^{2}\left(x, \eta_{+}^{*}\right) d x \\
& =\left(i \beta \int G^{2}\left(x, \eta_{+}^{*}\right) d x+\gamma_{\eta_{+}}-\frac{i \beta}{\tau^{*}}\right)\left(\hat{\eta}_{+}\right)^{2}
\end{aligned}
$$

which implies that

$$
\int|\hat{u}|^{2}+\left(\frac{1}{\tau^{*}}-\int G^{2}\left(x, \eta_{+}^{*}\right) d x\right)\left(\hat{\eta}_{+}\right)^{2}=0 .
$$

From $(12)$ and $(13) \frac{\mu+1}{\tau^{*}}\left(1+\rho_{0}^{2}\right)=\left(G\left(\eta_{+}^{*}, \eta_{+}^{*}\right)+\gamma_{\eta_{+}}\right)\left(1+\rho_{0}^{2}\right)$, which together with (15) implies that

$$
\int|\hat{u}|^{2}+\left(\frac{G\left(\eta_{+}^{*}, \eta_{+}^{*}\right)+\gamma_{\eta_{+}}}{\mu+1}-\int G^{2}\left(x, \eta_{+}^{*}\right) d x\right)\left(\hat{\eta}_{+}\right)^{2}=0 .
$$

This is equivalent to the equation

$$
\int|\hat{u}|^{2}+\frac{\gamma_{\eta_{+}}}{\mu+1}\left(\hat{\eta}_{+}\right)^{2}=0 .
$$

So we conclude that $\hat{u}=0$ and $\hat{\eta}_{+}=0$.

We have shown (8), and thus get a $C^{1}$-curve $\tau \mapsto(\phi(\tau), \lambda(\tau))$ of eigendata such that $\phi\left(\tau^{*}\right)=\phi^{*}$ and $\lambda\left(\tau^{*}\right)=i \beta$.

It remains to be shown that the transversality condition $\operatorname{Re} \lambda^{\prime}\left(\tau^{*}\right) \neq 0$ holds. Implicit differentiation of $E\left(\psi_{0}(\tau), \eta_{+}(\tau), \lambda(\tau), \tau\right)=0$ implies that

$$
\begin{aligned}
& D_{\left(u, \eta_{+}, \lambda\right)} E\left(\psi_{0}, \rho_{0}, i \beta, \tau^{*}\right)\left(\psi_{0}^{\prime}\left(\tau^{*}\right), \eta_{+}{ }^{\prime}\left(\tau^{*}\right), \lambda^{\prime}\left(\tau^{*}\right)\right) \\
& =\left(\begin{array}{c}
\left(\psi_{0}\left(\eta_{-}^{*}\right)+\gamma_{\eta_{+}}\left(\rho_{0}-1\right)\right) G\left(\cdot, \eta_{-}^{*}\right)+\left(\psi_{0}\left(\eta_{+}^{*}\right)+\gamma_{\eta_{+}}\left(\rho_{0}-1\right)\right) G\left(\cdot, \eta_{+}^{*}\right) \\
\psi_{0}\left(\eta_{-}^{*}\right)+\gamma_{\eta_{+}}\left(\rho_{0}-1\right) \\
\psi_{0}\left(\eta_{+}^{*}\right)+\gamma_{\eta_{+}}\left(\rho_{0}-1\right)
\end{array}\right) .
\end{aligned}
$$

This means that the function $\widetilde{u}:=\psi^{\prime}\left(\tau^{*}\right), \widetilde{\eta}_{+}:=\eta_{+}{ }^{\prime}\left(\tau^{*}\right)$ and $\widetilde{\lambda}:=\lambda^{\prime}\left(\tau^{*}\right)$ satisfy the equations

$$
\left\{\begin{array}{l}
(A+i \beta) \widetilde{u}+\widetilde{\lambda} \psi_{1}=-i \beta \widetilde{\eta}_{+} G\left(\cdot, \eta_{+}^{*}\right) \\
\widetilde{\lambda}-\tau^{*}\left(\widetilde{u}\left(\eta_{-}^{*}\right)+\gamma_{\eta_{+}} \widetilde{\eta}_{+}\right)=\psi_{0}\left(\eta_{-}^{*}\right)+\gamma_{\eta_{+}}\left(\rho_{0}-1\right) \\
-\widetilde{\lambda} \rho_{0}-i \beta \eta_{+}^{*}-\tau^{*}\left(\widetilde{u}\left(\eta_{+}^{*}\right)+\gamma_{\eta_{+}} \tilde{\eta}_{+}\right)=\psi_{0}\left(\eta_{+}^{*}\right)+\gamma_{\eta_{+}}\left(\rho_{0}-1\right)
\end{array}\right.
$$


where $\psi_{1}:=\psi_{0}-G\left(\cdot, \eta_{-}^{*}\right)+G\left(\cdot, \eta_{+}^{*}\right) \rho_{0}$. The equations (12) and (16) implies that

$$
\begin{aligned}
& \widetilde{u}\left(\eta_{-}^{*}\right)=\frac{\widetilde{\lambda}}{\tau^{*}}-\frac{i \beta}{\tau^{* 2}}-\gamma_{\eta_{+}} \widetilde{\eta}_{+} \\
& \widetilde{u}\left(\eta_{+}^{*}\right)=-\frac{\widetilde{\lambda} \rho_{0}}{\tau^{*}}-\frac{i \beta \eta_{+}^{*}}{\tau^{*}}+\frac{i \beta}{\left(\tau^{*}\right)^{2}} \rho_{0}-\gamma_{\eta_{+}} \widetilde{\eta}_{+} .
\end{aligned}
$$

We first show that $\widetilde{\lambda} \neq 0$. Suppose that $\widetilde{\lambda}=0$ in the system (16). Multiplying $\psi_{1}$ by (16) and $\widetilde{u}$ by (11) and then integrating,

$$
\begin{aligned}
\int_{\mathbb{R}}(A+i \beta) \widetilde{u} \psi_{1} & =\widetilde{\eta}_{+} \psi_{0}\left(\eta_{+}^{*}\right) \\
\int_{\mathbb{R}}(A+i \beta) \psi_{1} \widetilde{u} & =-\left(\widetilde{u}\left(\eta_{-}^{*}\right)-\widetilde{u}\left(\eta_{+}^{*}\right) \rho_{0}\right)
\end{aligned}
$$

which implies that

$$
\frac{i \beta}{\tau^{* 2}}\left(1+\rho_{0}^{2}\right)=\widetilde{\eta}_{+}\left(\psi_{0}\left(\eta_{+}^{*}\right)+\frac{i \beta}{\tau^{*}} \rho_{0}+\gamma_{\eta_{+}}\left(\rho_{0}-1\right)\right)=0 .
$$

This is a contradiction since $\beta\left(1+\rho_{0}^{2}\right) \neq 0$ and hence $\widetilde{\lambda} \neq 0$.

Multiplying $\widetilde{u}$ by (11) and integrating, and then comparing with (16), we obtain

$$
\widetilde{\lambda} \int_{\mathbb{R}} \psi_{1}^{2}+i \beta \widetilde{\eta}_{+} \int_{\mathbb{R}} G\left(x, \eta_{+}^{*}\right) \psi_{1}(x) d x=\widetilde{u}\left(\eta_{-}^{*}\right)-\widetilde{u}\left(\eta_{+}^{*}\right) \rho_{0} .
$$

Applying the (17), then we have

$$
\widetilde{\lambda}\left(\int\left(\psi_{1}^{2}-\left|\psi_{1}\right|^{2}\right)\right)=-\frac{i \beta}{\left(\tau^{*}\right)^{2}}\left(1+\rho_{0}^{2}\right)
$$

which implies that

$$
\operatorname{Re} \widetilde{\lambda}\left(\operatorname{Re} \int \psi_{1}^{2}-\int\left|\psi_{1}\right|^{2}\right)-\operatorname{Im} \widetilde{\lambda}\left(\operatorname{Im} \int \psi_{1}^{2}\right)=0
$$

and

$$
\operatorname{Im} \widetilde{\lambda}\left(\operatorname{Re} \int \psi_{1}^{2}-\int\left|\psi_{1}\right|^{2}\right)+\operatorname{Re} \widetilde{\lambda}\left(\operatorname{Im} \int \psi_{1}^{2}\right)=-\frac{\beta}{\left(\tau^{*}\right)^{2}}\left(1+\rho_{0}^{2}\right) .
$$

We now suppose that $\operatorname{Re} \widetilde{\lambda}=0$ in the equation (20). Then we have $\operatorname{Im} \int \psi_{1}^{2}=0$ which implies that $\operatorname{Re} \psi_{1}=0$ or $\operatorname{Im} \psi_{1}=0$. If $\operatorname{Im} \psi_{1}=0$ then $\operatorname{Re} \int \psi_{1}^{2}=\int\left|\psi_{1}\right|^{2}$ and thus from the equation $(21)$ we have $\frac{\beta}{\left(\tau^{*}\right)^{2}}\left(1+\rho_{0}^{2}\right)=0$. Therefore we have $\operatorname{Re} \psi_{1}=0$ and from the equation (12), we obtain

$$
G\left(\eta_{-}^{*}, \eta_{-}^{*}\right)\left(1+\rho_{0}^{2}\right)-2 G\left(\eta_{-}^{*}, \eta_{+}^{*}\right) \rho_{0}-\gamma_{\eta_{+}}\left(1-\rho_{0}\right)^{2}=0
$$

implies that $\left(G\left(\eta_{-}^{*}, \eta_{-}^{*}\right)-\gamma_{\eta_{+}}\right)\left(1+\rho_{0}^{2}\right)=0$ and this contradicts to Re $\psi_{1}=0$. Hence $\operatorname{Re} \widetilde{\lambda} \neq 0$ and is given by

$$
\left(\left(\operatorname{Re} \int \psi_{1}^{2}-\int\left|\psi_{1}\right|^{2}\right)^{2}+\left(\operatorname{Im} \int \psi_{1}^{2}\right)^{2}\right) \operatorname{Re} \widetilde{\lambda}=-\frac{\beta}{\left(\tau^{*}\right)^{2}}\left(1+\rho_{0}^{2}\right)\left(\operatorname{Im} \int \psi_{1}^{2}\right) .
$$

Hence the transversality condition holds.

We shall show that there exists a unique $\tau^{*}$ such that the linearization $-\widetilde{A}+\tau^{*} B$ has a purely imaginary pair of eigenvalues. To do this, we have to show that the function $\left(u, \eta_{+}, \beta, \tau\right) \mapsto E\left(u, \eta_{+}, i \beta, \tau\right)$ has a unique zero with $\beta>0$ and $\tau>0$. This means solving the system (7) with $\lambda=i \beta$ and $u=v+G\left(\cdot, \eta_{-}^{*}\right)-G\left(\cdot, \eta_{+}^{*}\right) \eta_{+}$,

$$
(A+i \beta) v=-\delta_{\eta_{-}^{*}}+\delta_{\eta_{+}^{*}} \cdot \eta_{+}
$$


and

$$
\begin{aligned}
\frac{i \beta}{\tau^{*}} & =v\left(\eta_{-}^{*}\right)+G\left(\eta_{-}^{*}, \eta_{-}^{*}\right)-G\left(\eta_{-}^{*}, \eta_{+}^{*}\right) \eta_{+}+\gamma_{\eta_{+}}\left(\eta_{+}-1\right) \\
-\frac{i \beta}{\tau^{*}} \eta_{+} & =v\left(\eta_{+}^{*}\right)+G\left(\eta_{+}^{*}, \eta_{-}^{*}\right)-G\left(\eta_{+}^{*}, \eta_{+}^{*}\right) \eta_{+}+\gamma_{\eta_{+}}\left(\eta_{+}-1\right) .
\end{aligned}
$$

The equation (22) has, for fixed $\beta \geq 0$, the unique solution $v=-G_{\beta}\left(\cdot, \eta_{-}^{*}\right)+$ $G_{\beta}\left(\cdot, \eta_{+}^{*}\right) \eta_{+}$where $G_{\beta}$ is the Green's function for the operator $A+i \beta$. The real and imaginary parts of the equation (23) and (24) imply that

$$
\left\{\begin{aligned}
& 0=-\operatorname{Re}\left(G_{\beta}\left(\eta_{-}^{*}, \eta_{-}^{*}\right)\left(1+\eta_{+}^{2}\right)-2 G_{\beta}\left(\eta_{-}^{*}, \eta_{+}^{*}\right) \eta_{+}\right)+G\left(\eta_{-}^{*}, \eta_{-}^{*}\right)\left(1+\eta_{+}^{2}\right) \\
&-2 G\left(\eta_{-}^{*}, \eta_{+}^{*}\right) \eta_{+}-\gamma_{\eta_{+}}\left(1-\eta_{+}\right)^{2} \\
& \frac{\beta}{\tau}\left(1+\eta_{+}^{2}\right)=-\operatorname{Im}\left(G_{\beta}\left(\eta_{-}^{*}, \eta_{-}^{*}\right)\left(1+\eta_{+}^{2}\right)-2 G_{\beta}\left(\eta_{-}^{*}, \eta_{+}^{*}\right) \eta_{+}\right) .
\end{aligned}\right.
$$

Lemma 4.3. Let $G_{\beta}$ be a Green function of the differential operator $A+i \beta$. Then the expression $\operatorname{Re}\left(G_{\beta}\left(\eta_{-}^{*}, \eta_{-}^{*}\right)\left(1+\eta_{+}^{2}\right)-2 G_{\beta}\left(\eta_{-}^{*}, \eta_{+}^{*}\right) \eta_{+}\right)$is strictly decreasing in $\beta \in \mathbb{R}^{+}$with

$$
\operatorname{Re} G_{0}\left(\eta_{ \pm}^{*}, \eta_{ \pm}^{*}\right)=G\left(\eta_{ \pm}^{*}, \eta_{ \pm}^{*}\right), \lim _{\beta \rightarrow \infty} \operatorname{Re} G_{\beta}\left(\eta_{ \pm}^{*}, \eta_{ \pm}^{*}\right)=0
$$

and $\operatorname{Im}\left(G_{\beta}\left(\eta_{-}^{*}, \eta_{-}^{*}\right)\left(1+\eta_{+}^{2}\right)-2 G_{\beta}\left(\eta_{-}^{*}, \eta_{+}^{*}\right) \eta_{+}\right)<0$ for any $\beta>0$.

Proof. First we have $(A+i \beta)^{-1}=(A-i \beta)\left(A^{2}+\beta^{2}\right)^{-1}$, so if $L(\beta):=\operatorname{Re}(A+$ $i \beta)^{-1}$ and $T(\beta):=\operatorname{Im}(A+i \beta)^{-1}$, then

$$
L(\beta)=A\left(A^{2}+\beta^{2}\right)^{-1} \text { and } T(\beta)=-\beta\left(A^{2}+\beta^{2}\right)^{-1} .
$$

Since $\left(A^{2}+\beta^{2}\right)^{-1}$ is a positive operator, it follows that $-T(\beta)$ is positive for $\beta>0$, which implies that $\operatorname{Im}\left(G_{\beta}\left(\eta_{-}^{*}, \eta_{-}^{*}\right)\left(1+\eta_{+}^{2}\right)-2 G_{\beta}\left(\eta_{-}^{*}, \eta_{+}^{*}\right) \eta_{+}\right)<0$. Moreover, $L(\beta) \longrightarrow A^{-1}$ as $\beta \rightarrow 0$ and $L(\beta) \longrightarrow 0$ as $\beta \rightarrow \infty$, which results in the corresponding limiting behavior for $\operatorname{Re}\left(G_{\beta}\left(\eta_{ \pm}^{*}, \eta_{ \pm}^{*}\right)\right)$.

Now to show that $\beta \mapsto \operatorname{Re}\left(G_{\beta}\left(\eta_{-}^{*}, \eta_{-}^{*}\right)\left(1+\eta_{+}^{2}\right)-2 G_{\beta}\left(\eta_{-}^{*}, \eta_{+}^{*}\right) \eta_{+}\right)$is strictly decreasing, define $h(\beta)(x):=G_{\beta}\left(x, \eta_{-}^{*}\right)-G_{\beta}\left(x, \eta_{+}^{*}\right) \eta_{+}-G\left(x, \eta_{-}^{*}\right)+G\left(x, \eta_{+}^{*}\right) \eta_{+}$. Then (in the weak sense at first)

$$
(A+i \beta) h(\beta)=-i \beta\left(G\left(\cdot, \eta_{-}^{*}\right)-G\left(\cdot, \eta_{+}^{*}\right) \eta_{+}\right) .
$$

As a result $h(\beta) \in D(A)_{\mathbf{C}}$ and $h: \mathbb{R}^{+} \rightarrow D(A)_{\mathbf{C}}$ is differentiable with $i h(\beta)+(A+$ $i \beta) h^{\prime}(\beta)=-i\left(G\left(\cdot, \eta_{-}^{*}\right)-G\left(\cdot, \eta_{+}^{*}\right) \eta_{+}\right)$, therefore

$$
(A+i \beta) h^{\prime}(\beta)=-i\left(G_{\beta}\left(\cdot, \eta_{-}^{*}\right)-G_{\beta}\left(\cdot, \eta_{+}^{*}\right) \eta_{+}\right) .
$$

We thus get

$$
\begin{aligned}
& -i\left(\overline{h^{\prime}(\beta)\left(\eta_{-}^{*}\right)}-\overline{h^{\prime}(\beta)\left(\eta_{+}^{*}\right) \eta_{+}}\right) \\
= & \int_{\mathbb{R}}(A+i \beta)^{2} h^{\prime}(\beta) \overline{h^{\prime}(\beta)(x)} d x \\
= & \int_{\mathbb{R}}(A+i \beta) h^{\prime}(\beta) \cdot(A+i \beta) \overline{h^{\prime}(\beta)} d x \\
= & \int_{\mathbb{R}}\left|A h^{\prime}(\beta)\right|^{2}-\beta^{2}\left|h^{\prime}(\beta)\right|^{2} d x+2 i \beta \int_{\mathbb{R}} A h^{\prime}(\beta) \overline{h^{\prime}(\beta)} d x .
\end{aligned}
$$


It follows that

$$
\operatorname{Re}\left(h^{\prime}(\beta)\left(\eta_{-}^{*}\right)-h^{\prime}(\beta)\left(\eta_{+}^{*}\right) \eta_{+}\right)=-2 \beta \int_{\mathbb{R}}\left|A^{1 / 2} h^{\prime}(\beta)\right|^{2}<0
$$

and thus

$$
\operatorname{Re}\left(h^{\prime}(\beta)\left(\eta_{-}^{*}\right)-h^{\prime}(\beta)\left(\eta_{+}^{*}\right) \eta_{+}\right)=\operatorname{Re}\left(G_{\beta}^{\prime}\left(\eta_{-}^{*}, \eta_{-}^{*}\right)\left(1+\eta_{+}^{2}\right)-2 G_{\beta}^{\prime}\left(\eta_{-}^{*}, \eta_{+}^{*}\right) \eta_{+}\right)<0 .
$$

Theorem 4.4. Assume $0<\frac{1}{2}-a_{0}+\frac{\mu \kappa}{\mu+1}<\frac{1}{2(\mu+1)}$, then for a unique critical point $\tau^{*}>0$, there exists a unique, purely imaginary eigenvalue $\lambda=i \beta$ of (7) with $\beta>0$.

Proof. From the equation (25) we now define

$$
\begin{aligned}
K(\beta):= & -\operatorname{Re}\left(G_{\beta}\left(\eta_{-}^{*}, \eta_{-}^{*}\right)\left(1+\eta_{+}^{2}\right)-2 G_{\beta}\left(\eta_{-}^{*}, \eta_{+}^{*}\right) \eta_{+}\right)+G\left(\eta_{-}^{*}, \eta_{-}^{*}\right)\left(1+\eta_{+}^{2}\right) \\
& -2 G\left(\eta_{-}^{*}, \eta_{+}^{*}\right) \eta_{+}-\gamma_{\eta_{+}}\left(1-\eta_{+}\right)^{2} .
\end{aligned}
$$

Then we have $K(0)=-\gamma_{\eta_{+}}\left(1-\eta_{+}\right)^{2}<0$ and $\lim _{\beta \rightarrow \infty} K(\beta)=G\left(\eta_{-}^{*}, \eta_{-}^{*}\right)\left(1+\eta_{+}^{2}\right)-$ $2 G\left(\eta_{-}^{*}, \eta_{+}^{*}\right) \eta_{+}-\gamma_{\eta_{+}}\left(1-\eta_{+}\right)^{2}=\left(G\left(\eta_{-}^{*}, \eta_{-}^{*}\right)-\gamma_{\eta_{+}}\right)\left(1+\eta_{+}\right)^{2}>0$. Moreover, $K^{\prime}(\beta)>$ 0 by Lemma 4.3. Therefore, there exists a unique $\beta$ and from this $\beta$ the unique $\tau^{*}>0$ can be found using (25).

The main result of effects on the inhomogeneity states as follows:

Theorem 4.5. Assume that $0<\frac{1}{2}-a_{0}+\frac{\mu \kappa}{\mu+1}<\frac{1}{2(\mu+1)}$. Then (3), respectively (2), has at least one stationary solutions $\left(u^{*}, \eta_{-}^{*}, \eta_{+}^{*}\right)$ where $u^{*}=0$, respectively $\left(v^{*}, \eta_{-}^{*}, \eta_{+}^{*}\right)$ for all $\tau$. Then there exists a unique $\tau^{*}$ such that the linearization $-\widetilde{A}+\tau^{*} B$ has a purely imaginary pair of eigenvalues. The point $\left(0, \eta_{-}^{*}, \eta_{+}^{*}, \tau^{*}\right)$ is then a Hopf point for (3) and there exists a $C^{0}$-curve of nontrivial periodic orbits for (3), respectively (2), bifurcating from $\left(0, \eta_{-}^{*}, \eta_{+}^{*}, \tau^{*}\right)$, respectively $\left(v^{*}, \eta_{-}^{*}, \eta_{+}^{*}, \tau^{*}\right)$.

\section{References}

1. S. Bouzat and H.S. Wio, Pattern dynamics in inhomogeneous active media, Physica A 293 (2001), 405-420.

2. P. Fife and J. Tyson, Target pattern in a realistic model of Belousov-Zhabotinskii reaction, J, Chem. Phys. 73 (1980), 2224-2237.

3. A. V. Gorbatyuk and P. B. Rodin, Current filamentation in bistable semiconductor systems with two global constraints, Z. Phys. B: Condens. Matter 104 (1997), 45-54.

4. Y.M.Ham-Lee, R.Schaaf and R.Thompson, A Hopf bifurcation in a parabolic free boundary problem, J. of Comput. Appl. Math. 52 (1994), 305-324.

5. A.L. Hodgkin and A. F. Huxley, A qualitative description of membrane current and its application to conduction and excitation in nerve, J. Physiol. 117 (1951), 500-514.

6. H. Ikeda and M. Mimura, Wave-blocking phenomena in bistable reaction-diffusion systems, SIAM J. Appl. Math. 49 (1989), 515-538.

7. H. Ikeda and M. Mimura, Stability analysis of stationary solutions of bistable reaction-variable diffusion systems, SIAM J. Math. Anal. 22 (1991), 1651-1678.

8. J. P. Keener, A geometrical theory for spiral waves in excitable media, SIAM J. Appl. Math. 46, (1986), 1039-1056.

9. S. Koga, Y. Kuramoto, Localized Patterns in Reaction-Diffusion Systems, Prog. Theor. Phys. 63 (1980), 106-121.

10. Y-X Li, Tango waves in a bidomain model of fertilization calcium waves, Physica D 186 (2003) 27-89. 
11. H.P. McKean, Nagumo's equation, Adv. Math. 4 (1975), 209-223.

12. Meinhardt, Models of Biological Pattern Formulation, Academic Press, London, 1982.

13. M. Mimura, M. Tabata and Y. Hosono, Multiple solutions of two-point boundary value problems of Neumann type with a small parameter, SIAM J. Math. Anal. 11 (1981), 613-633.

14. Y. Nishiura and H. Fujii, Stability of singularly perturbed solutions to systems of reactiondiffusion equations, SIAM J. Math. Anal. 18 (1987), 1726-1770.

15. Y. Nishiura and M. Mimura, Layer oscillations in reaction-diffusion systems, SIAM J. Appl. Math. 49 (1989), 481-514.

16. T. Ohta, A. Ito and A Tetsuka, Self-organization in an excitable reaction-diffusion system: Synchronization of oscillatory domains in one dimension, Phys. Rev. A. 42 (1990), 3225-3.

17. T. Ohta, M. Mimura, and R. Kobayashi, Higher dimensional localized patterns in excitable media, Phys. D 34 (1989), 115-144.

18. J.P. Pauwelussen, Nerve impulse propagation in a branching nerve system: a simple model, Physica D 4 (1981), 67-88.

19. A. Prat and Y-X. Li, Stability of front solutions in inhomogeneous media, Physica D 186 (2003) 50-68.

20. K. Sakamoto, Spatial homogenization and internal layers in a reaction-diffusion system, Hiroshima Math. J, 30 (200), 377-402.

21. P. Schutz, M. Bode, and H-G Purwins, Bifurcations of front dynamics in a reaction diffusion system with spatial inhomogeneities, Physica D 82 (1995) 382-397.

22. M. Suzuki, T. Ohta, M. Mimura and H. Sakaguchi, Breathing and wiggling motions in three species laterally inhibitory systems, Phys. Rev. E, 52 (1995), 3645-3655.

23. A. T. Winfree, The geometry of Biological time, Biomathematics 8, Springer, Berlin, 1980.

24. E. Yanagida, Stability of stationary distributions in a space-dependent population growth process J. Math. Biol. 15 (1982), 37-50.

Department of Mathematics, Kyonggi University, Suwon, 442-760, Korea

E-mail address: ymham@kyonggi.ac.kr 